\title{
Uso de las tecnologías de la información y la comunicación como competencia transversal en la formación inicial de docentes
}

\author{
Use of Information and Communication Technologies as a Transversal Competence in \\ Teacher Training
}
Uso das tecnologias da informação e comunicação como competência transversal na formação inicial de docentes

Sandra Araceli Varela-Ordorica

Tecnologico de Monterrey Monterrey, México sandra8488@hotmail.com iD https://orcid.org/0000-0001-9994-3209 Jaime Ricardo Valenzuela-González

Tecnologico de Monterrey Monterrey, México jrvg@tec.mx

https://orcid.org/0000-0003-3999-501X

\author{
Recibido • Received • Recebido: 02 / 05 / 2018 \\ Corregido • Revised • Revisado: 23 / 09 / 2019 \\ Aceptado • Accepted $\cdot$ Aprovado: 07 / $11 / 2019$
}

\begin{abstract}
Resumen: El objetivo de esta investigación fue explorar cómo los maestros y maestras promueven el desarrollo de la competencia transversal uso de las tecnologías de la información y la comunicación (TIC) en el futuro personal docente de educación primaria. El estudio se llevó a cabo en una escuela normal rural del Estado de México, usando el método de estudio de casos. Se realizaron entrevistas a trece docentes, así como observaciones a sus clases impartidas. El análisis de datos se realizó con el programa Atlas.ti. Entre los hallazgos destaca que el uso de las TIC, por parte del profesorado, propicia que el estudiantado normalista se motive a emplearlas, no solo como medio de entretenimiento o socialización, sino como un recurso didáctico para aprender y enseñar temas relacionados con la educación de primaria; se destaca que el uso de las TIC vuelve las clases más llamativas, dinámicas y claras; favorece la interacción social, la investigación en Internet, el aprendizaje autorregulado y el desarrollo de redes para aprender. El estudiantado participante en este estudio demostró el logro de esta competencia en la elaboración de sus trabajos académicos, búsquedas de información en internet, aplicación de software educativo para aprender matemáticas, uso de redes sociales y diseño de blogs. Asimismo, se encontró que, no obstante, las políticas educativas a nivel nacional contemplan como meta la alfabetización digital de niños, niñas y jóvenes, en realidad no siempre se cuenta en las escuelas de educación básica y normal con las condiciones y recursos tecnológicos para que tanto docentes como estudiantes adquieran, en forma óptima, las competencias digitales necesarias para desenvolverse mejor en una sociedad basada en el conocimiento.
\end{abstract}

Palabras claves: Habilidades digitales; competencia transversal; tecnologías de la información y la comunicación; formación inicial de docentes. 
doi: http://doi.org/10.15359/ree.24-1.10

URL: http://www.una.ac.cr/educare

CORREO: educare@una.cr

\begin{abstract}
The purpose of this research study was to learn how teachers promote the development of the transversal competence use of information and communication technologies (ICT) in future teachers of elementary education. The study took place in a teacher education school of the State of Mexico, using the case study method. Thirteen teachers were interviewed, as well as observations were made in their classes. Data analysis was done with the Atlas.ti program. In this study, it was found that the use of ICT by teachers encourages the students to be willing to use such technologies, not only as a mean of entertainment or socialization, but as a teaching resource to learn how to teach subjects related to the education of children in elementary school. The use of ICT makes classes more attractive, dynamic, and understandable; it promotes social interaction, the use of Internet for research purposes, self-regulated learning, and the development of learning networks. Students participating in this study demonstrated the achievement of this competence in the elaboration of their academic papers, the search for information on Internet, the use of educational software to learn math, the use of social networks, and blog design. It was found that, in spite that Mexico educational policies have, as a goal, the digital literacy of children and young people, actually, elementary and teacher education schools do not always have the conditions and technological resources to allow that both teachers and students develop the digital competences for their success in a knowledgebased society.
\end{abstract}

Keywords: Digital skills; transversal competence; information and communication technologies; teacher education.

Resumo: O objetivo desta investigação foi explorar a forma como professoras e professores promovem o desenvolvimento da competência transversal, uso das tecnologias de informação e comunicação (TIC) no futuro pessoal docente de educação primária. A pesquisa foi realizada em uma escola normal rural do Estado do México, usando o método de estudo de casos. Foram realizadas entrevistas a treze professores, como também observações da forma como lecionam. A análise de dados é realizada com o programa Atlas.ti. Entre os resultados obtidos, se destaca que o uso das TICs por parte dos professores propicia que pessoas estudantes normalistas se sintam motivadas a utiliza-las, não somente como meio de entretenimento ou socialização, mas também como um recurso didático para aprender e compartilhar temas relacionados com o ensino fundamental; Destaca-se que uso de TIC torna as classes mais atraentes, dinâmicas e claras; favorece a interação social, a investigação na Internet, o aprendizado autorregulado e o projeto de redes para aprender. Estudantes participantes desta pesquisa demostraram o resultado desta competência na elaboração de seus trabalhos acadêmicos, buscando informações na internet, aplicação de software educacional para aprender matemática, uso de redes sociais e projetos de blogs. Também foi encontrado que, apesar das políticas educacionais a nível nacional contemplarem como meta a alfabetização digital das crianças e jovens, na realidade, nem sempre as escolas de ensino fundamental contam com as condições e recursos tecnológicos para que, tanto docentes como estudantes consigam, de forma óptima, as competências digitais necessárias para desenvolver-se melhor em uma sociedade baseada no conhecimento.

Palavras chave: Habilidades digitais; competência transversal; tecnologias de informação e comunicação; formação inicial de docentes. 


\section{Introducción}

\section{Las tecnologías de la información y la comunicación (TIC) como competencia transversal para una sociedad basada en el conocimiento}

En la formación inicial y continua de docentes de educación básica, una de las necesidades actuales es el desarrollo y fortalecimiento de competencias transversales en la aplicación de las TIC en la práctica educativa. El siglo XXI implica el reto de usar estrategias de enseñanza innovadoras centradas en el estudiantado. En concreto, el personal docente debe incorporar recursos tecnológicos para favorecer habilidades intelectuales, sobre todo el autoaprendizaje y que, al mismo tiempo, propicien un desarrollo integral más amplio.

El desarrollo de competencias transversales en el uso de las TIC se ve influenciado por los vertiginosos cambios que se dan a nivel mundial en la educación (Mortera, 2016), así como en los ámbitos económicos, políticos y sociales. La educación ha de transformarse para estar a la altura de los retos que implican los acontecimientos de orden global, las nuevas economías, los adelantos científicos y tecnológicos en todos los órdenes del quehacer humano. Por este motivo, organismos como la Organización de las Naciones Unidas para la Educación, la Ciencia y la Cultura (UNESCO, 2008, 2009) y la Secretaría de Educación Pública (SEP, 2012), han implementado programas que contemplan el desarrollo de competencias transversales, entre las que se encuentra el uso de las TIC.

A fin de tener mayor precisión en el análisis de las TIC, como una de las competencias transversales para una sociedad del conocimiento, es necesario abordar dos conceptos: competencia y transversalidad.

Primeramente, hay que tener claridad sobre lo que es una competencia. De acuerdo con Valenzuela (2016), "el concepto de competencia ... está estrechamente relacionado con desempeño" (p. 1), el mismo que debe ser observable para inferir en qué medida se logró el nivel de conocimientos, habilidades o aptitudes deseado.

El enfoque de una educación basada en competencias se ubica dentro de la ideología de la eficiencia social (Schiro, 2008), que parte de la premisa de que "el propósito de las instituciones educativas es satisfacer eficientemente las necesidades de la sociedad, en especial las del sector productivo" (p. 5). Al respecto, el objetivo del proceso educativo es, precisamente, que un sujeto principiante pase a ser un sujeto experto, mediante las experiencias adquiridas, no solo por la cantidad de estas, sino también por su calidad; experiencias que permitan al individuo atender tanto los requerimientos de las "sociedades del conocimiento"; como aprender a lo largo de la vida, convivir en armonía con las demás personas, cuidar su entorno natural, usar las TIC, dentro y fuera de la escuela (UNESCO, 2009). 
doi: http://doi.org/10.15359/ree.24-1.10

URL: http://www.una.ac.cr/educare

CORREO: educare@una.cr

El otro concepto por precisar es el de transversalidad. En el contexto del diseño curricular, de acuerdo con Valenzuela (2016),"por una parte se refiere al diseño de cursos multidisciplinarios y de proyectos interdisciplinarios; por otra parte, se emplea para adjetivar el concepto de competencia" (p. 9), en este sentido, las competencias transversales son aquellas que están presentes en todas o la mayoría de las asignaturas o cursos de un programa de estudios. El uso de las TIC es una de las competencias transversales incluidas en los planes y programas de estudios de licenciatura y de posgrado de muchos países.

\section{Las TIC en la enseñanza}

En los últimos años se ha presentado un incremento a nivel global del uso de diversas tecnologías de la información en la enseñanza; sin embargo, en las escuelas aún se aprecia cierto rezago o resistencia a la utilización de las TIC como herramientas de enseñanza-aprendizaje y su uso no es significativo (Cuban, 2001), no obstante, el acelerado ritmo en que ocurren las transformaciones tecnológicas. Numerosos estudios alrededor del mundo (España: Suárez, Almerich, Gargallo y Aliaga, 2010; Bélgica: Valcke, Rots, Verbeke y van Braak, 2007; Singapur: Chai, Koh y Tsai, 2013) dan cuenta de cómo las creencias del profesorado respecto al uso de las TIC en la educación, los factores culturales y contextuales, así como las políticas educativas, inciden de manera determinante en la incorporación o no de estas a la enseñanza.

Mortera (2016) refiere que "las habilidades del maestro en el manejo de las TIC y las pedagogías apropiadas son fundamentales para su integración exitosa al proceso de enseñanzaaprendizaje" (p. 131). Por su parte, Suárez et al. (2010), en una investigación realizada con profesores y profesoras de centros de primaria y secundaria de Valencia, España, encontraron que el profesorado, por su falta de competencia en su uso de las TIC con fines pedagógicos, no se sentía confiado.

Una mejor preparación del personal docente previa a la incorporación de las tecnologías en el aula resultará beneficiosa tanto para este como para sus estudiantes Mortera (2016), puesto que la enseñanza no es eficaz si no se sabe cómo utilizar adecuadamente las TIC como recurso didáctico, además de emplearlas para otros fines.

Suárez, Almerich, Orellana y Belloch (2012), en un estudio que realizaron con el propósito de establecer un modelo básico del uso de las TIC, tanto en el plano personal-profesional como en el aula con estudiantes, encontraron que el profesorado utiliza las TIC principalmente para tareas administrativas y preparación de sus clases, con muy escaso uso en el aula, en recursos multimedia, presentaciones y software educativo.

Las competencias tecnológicas inciden en forma significativa en las competencias pedagógicas relacionadas con la aplicación en las TIC en la práctica educativa (Suárez, Almerich, Gargallo y Aliaga, 2013). La facilidad de acceso a las computadoras y la red de internet en un centro educativo favorecen que tanto docentes como estudiantes desarrollen más fácilmente competencias en su uso. 
Sin embargo, no es únicamente la facilidad de acceso a las TIC lo que determina o no su uso en la educación. Las creencias y las actitudes del equipo de docentes también son factores fundamentales (Ertmer, Ottenbreit-Leftwich, Sadik, Sendurur y Sendurur, 2012). El profesorado es consciente de que las TIC son una realidad y que, si no se prepara, quedará desfasado; sin embargo, aun cuando la mayoría vincula la innovación educativa a las TIC, no creen necesario utilizarlas para enseñar su materia (Orellana, Almerich, Belloch y Díaz, 2004).

\section{Influencia de las creencias sobre el uso de las TIC del profesorado en la incorporación de estas en el proceso educativo}

En un estudio realizado por Ertmer et al. (2012) con equipos docentes reconocidos en el uso de la tecnología, estos señalaron que lo que favorecía o impedía que otros maestros y otras maestras hicieran uso de la tecnología, eran sus actitudes y creencias hacia esta, así como sus actuales niveles de conocimientos y habilidades.

Se estima necesario que, previamente a incorporar las TIC en el aula, se lleven a cabo programas de fortalecimiento tecnológico y pedagógico para docentes con la finalidad de que adquieren mayores conocimientos y habilidades en el uso de las TIC con fines educativos, lo que contribuirá a aumentar su confianza y reducir el temor que les ocasiona su uso al educar a sus estudiantes (Almerich, Suárez-Rodríguez, Belloch y Bo, 2011). Si bien no es suficiente saber utilizar las computadoras, sí es uno de los pasos principales para incorporarlas en la enseñanza, por ello, un programa de formación integral en TIC debiera incluir conocimientos tecnológicos y pedagógicos sobre el uso de las TIC.

Tondeur, Valcke y van Braak (2008) analizaron la relación entre las creencias educativas, perfil de docentes (tradicional, constructivista) y su enfoque típico de uso de la computadora en el aula, y concluyeron que las creencias educativas de estos perfiles afectan las decisiones relacionadas con los diferentes tipos de uso que le dan a las computadoras en el aula.

\section{Necesidades formativas del profesorado para el uso de las TIC en la educación}

Con la finalidad de lograr mejores resultados en el aprendizaje del alumnado, las instituciones educativas buscan innovadoras estrategias de enseñanza. Ante esto, el papel del personal docente cobra mayor importancia como agente de cambio, por lo que es sumamente importante identificar cuáles son las competencias que deben desarrollar ante estos nuevos retos. Almerich et al. (2011) realizaron un estudio con 868 docentes de primaria y secundaria de Valencia, España, respecto al modelo de necesidades formativas que mejor se ajusta a sus necesidades reales para la formación e integración de las TIC en la práctica docente. Encontraron cuatro perfiles diferenciados: inicial, inicial-medio, medio y avanzado. 
doi: http://doi.org/10.15359/ree.24-1.10

URL: http://www.una.ac.cr/educare

CORREO: educare@una.cr

Los resultados muestran, en primer lugar, que el conjunto del profesorado presenta un conocimiento de los distintos recursos tecnológicos con algunas carencias; pero ningún grupo logran un conocimiento avanzado. Los recursos tecnológicos en los que demuestran mayor conocimiento son la gestión del sistema operativo; el procesador de texto; internet como forma de obtención de información e internet como forma de comunicación, principalmente el correo electrónico. Ello corresponde con los niveles de competencias menos avanzados indicados por la Organización de las Naciones Unidas para la Educación, la Ciencia y la Cultura (UNESCO, 2008).

Las competencias tecnológicas con el uso de las TIC por el profesorado, tanto para su uso personal como con fines pedagógicos en el aula son aspectos fundamentales para conseguir la confianza necesaria para la integración de las TIC en la educación (Almerich, Suárez-Rodríguez, Belloch y Orellana, 2010).

Docentes líderes, cuyas prácticas pedagógicas son más constructivistas que las de otros grupos de profesorado de la misma materia y nivel, utilizan las computadoras substancialmente más tiempo y en diversos procesos, esto es, existe una fuerte conexión entre liderazgo docente, uso sofisticado de las computadoras y uso de la tecnología en la formación docente (Riel y Becker, 2008).

Becker y Ravitz (2001), en respuesta a las ideas expuestas por Cuban (2001) respecto a que las computadoras no han transformado las prácticas de enseñanza de la mayoría del profesorado, refieren que, en cierto sentido, es correcta la idea de Cuban (2001); sin embargo, en las condiciones adecuadas, donde el equipo docente cuenta con los recursos tecnológicos, las mejores condiciones de trabajo en el aula y se basan en la pedagogía constructivista, tanto maestros y maestras como estudiantes muestran interés en su uso y tienen un impacto favorable, no solo en el rendimiento académico del estudiantado, sino fuera de clase también.

Respecto a las cualidades o características deseables necesarias del profesorado para aprovechar los recursos tecnológicos como herramientas pedagógicas significativas, en un estudio realizado por Ertmer y Ottenbreit-Leftwich (2010), centrado en cuatro variables sobre el personal docente como agente de cambio: el conocimiento, la autoeficacia, creencias pedagógicas y la cultura escolar disciernen sobre la influencia de las creencias de del profesorado en la aplicación de las TIC. La forma de pensar de estos grupos profesionales debe cambiar para comprender que la enseñanza no es eficaz sin el uso apropiado de las TIC para facilitar el aprendizaje del estudiantado (Ertmer y Ottenbreit-Leftwich, 2010). Una vez que se comprenda que la enseñanza no es eficaz sin el uso adecuado de los recursos TIC, se tendrá un logro importante para obtener buenos resultados en el aprendizaje estudiantil.

También es importante considerar la parte institucional (Mortera, 2016), puesto que las políticas educativas deben estar alineadas con las creencias de docentes respecto a la utilidad de las TIC para favorecer aprendizajes significativos en sus estudiantes (Tondeur et al., 2008). Una innovación es menos probable que se adopte, si no está acorde con los valores pedagógicos

6

Sandra Araceli Varela-Ordorica y Jaime Ricardo Valenzuela-González

Los artículos de la Revista Electrónica Educare del Centro de Investigación y Docencia en Educación de la Universidad Nacional, Costa Rica, se comparten bajo términos de la Licencia Creative Commons: Reconocimiento, No Comercial, Sin Obra Derivada 3.0 Costa Rica. Las autorizaciones adicionales a las aquí delimitadas se pueden obtener en el correo: educare@una.cr 
predominantes, las creencias y las prácticas de docentes y personal directivo de la escuela (Ertmer, 2005). Así mismo, la interacción con colegas con más experiencia en el manejo de la tecnología tiene un efecto positivo en su adopción por parte del resto (Ertmer, 2005).

\section{Las TIC en la formación inicial de docentes}

En México las políticas educativas contemplan la incorporación de las TIC en todos los niveles educativos (SEP, 2012), para lo cual resulta necesaria la preparación del futuro personal docente. Valcke et al. (2007) encontraron que las necesidades individuales de los maestros y maestras y las necesidades de la escuela son fundamentales a la vista de la validez del contenido y del diseño de los programas de su formación.

Así mismo, Tondeur et al. (2011) realizaron investigaciones cualitativas en Estados Unidos, Reino Unido, Turquía, Taiwán, Finlandia y Chipre, en torno a la formación inicial del profesorado para la integración de la tecnología en sus futuras aulas. Concluyen que es necesaria una mejor preparación del personal docente en formación para que utilice la tecnología en sus prácticas de enseñanza.

Se requiere dar más seguimiento al modelo de formación de maestros y maestras y atender la naturaleza de sus necesidades de formación (Tondeur et al., 2011); así mismo, analizar cómo las tecnologías Web 2.0, como blogs y wikis habilitados por muchos profesores y profesoras, las modificaciones a los planes de desarrollo y la integración de la tecnología en entornos de aprendizaje centrados en el estudiantado, pueden mejorar significativamente el aprendizaje.

Por otra parte, Koh y Divaharan (2011) y Koh, Chai y Tsai (2014) examinaron los resultados obtenidos con la aplicación del modelo instruccional TPACK en la formación de docentes, e identificaron siete tipos de conocimiento: conocimiento tecnológico (CT), conocimiento pedagógico $(\mathrm{PK})$, conocimiento de contenidos (CK), conocimiento tecnológico del contenido (TCK), conocimiento tecnológico pedagógico (TPK), conocimiento de contenido pedagógico (PCK) y conocimiento pedagógico tecnológico del contenido (TPACK).

Estos siete constructos caracterizan las diferentes necesidades de conocimiento del personal docente para la integración tecnológica, desde un enfoque constructivista de aprendizaje; sin embargo, no hay que olvidar que la actualización de los grupos formadores de docentes es esencial para que los futuros profesorados se apropien de las tecnologías, y se debe tener en cuenta que sus creencias y actitudes, así como los conocimientos que poseen y su capacidad para diseñar prácticas educativas innovadoras (Mortera, 2016) serán determinantes en la incorporación con éxito de las TIC en el aula.

La aceptación de la tecnología por parte de los futuros cuerpos docentes, así como sus actitudes hacia su uso, la utilidad percibida, la facilidad de uso observada y el disfrute al usarla para su desarrollo personal y profesional, son indicadores de la intención de usar las TIC en sus prácticas educativas (Teo y Noyes, 2011). 
doi: http://doi.org/10.15359/ree.24-1.10

URL: http://www.una.ac.cr/educare

CORREO: educare@una.cr

\section{Planteamiento del problema}

En el contexto mundial, la Organización de las Naciones Unidas para la Educación la Ciencia y la Cultura (UNESCO, 2008, 2009) ha realizado numerosos estudios para determinar los niveles de economía, salud, educación, así como su impacto en el crecimiento económico y en el mejoramiento de la calidad de vida de las personas. Propone políticas educativas que contribuyan a mejorar su desarrollo económico y, como consecuencia, las condiciones de vida de la población. Entre estas políticas se incluye en el uso de las TIC en la educación formal, en forma presencial y a distancia.

En México, a pesar de los esfuerzos del gobierno federal por dotar de infraestructura y equipos de cómputo a las escuelas primarias, no se han logrado transformar las prácticas de enseñanza de la mayoría del profesorado. Como lo mencionan Cuban (2001), Ertmer et al. (2012), no es suficiente contar con los recursos tecnológicos para tener buenos resultados. Es necesario saber utilizar apropiadamente la tecnología, además de considerar otros factores como las actitudes y creencias de docentes y estudiantes.

Las instituciones educativas deben prepararse para las transformaciones de orden mundial, donde son prioritarias las innovaciones en educación y el fortalecimiento de las competencias transversales que permitan a los futuros grupos docentes desenvolverse con éxito durante su formación inicial y en su desempeño como personal de primaria al egresar. Para ello es importante tratar de dar respuesta a la siguiente pregunta general de investigación:

¿Cómo el profesorado de educación normal hace uso de las TIC en su trabajo docente y de qué manera contribuye a desarrollar en sus estudiantes la competencia transversal: uso de las TIC?

Y a las siguientes preguntas subordinadas:

1. ¿Qué actitud manifiesta el estudiantado normalista cuando sus docentes emplean las TIC en sus prácticas pedagógicas, para contribuir al desarrollo de la competencia transversal uso de las TIC?

2. ¿Cómo el estudiantado normalista hace evidente el desarrollo de la competencia transversal uso de las TIC?

Numerosos estudios alrededor del mundo dan cuenta de la importancia de las TIC en la educación y de cómo diversos factores inciden en su aplicación o no en los procesos educativos. Como muestra de ello se pueden mencionar las siguientes investigaciones: Suárez et al. (2013) realizaron una investigación, en Valencia, España, titulada "Las competencias del profesorado en TIC: estructura básica", de corte cuantitativo, con el objetivo de establecer un modelo de estructuración y relación de las competencias en TIC para el profesorado. 
El objetivo general del estudio fue explorar de qué manera las personas formadoras de docentes hacen uso de las TIC en sus intervenciones pedagógicas y desarrollan en el estudiantado normalista la competencia transversal uso de las TIC, como medio de aprendizaje de contenidos disciplinares y como recurso didáctico para aplicarlo en las prácticas pedagógicas que realizan en las escuelas primarias.

En la formación inicial de docentes es necesario fortalecer la competencia del uso de las TIC en la educación, dentro y fuera del contexto escolar, para mejorar el desempeño académico del alumnado y prepararlo como docente de primaria, en donde, a su vez, favorecerá en sus estudiantes las competencias en el uso de las TIC.

El futuro de toda nación se sustenta, en gran medida, en su educación. Es indispensable replantear las políticas educativas que permitan a la ciudadanía estar acorde con las demandas de un mundo globalizado y cada vez más competitivo; por ello, los gobiernos de los países buscan alternativas para ofrecer una mejor calidad educativa, incluyente, innovadora, que permita potencializar los conocimientos y las virtudes de sus habitantes.

La UNESCO, como resultado de los numerosos estudios que ha realizado en el ámbito de educación a nivel mundial, ha establecido estándares de competencias para lograr una educación de calidad a lo largo de la vida. Argumenta que"el uso de las TIC en educación puede ampliar el acceso a oportunidades de aprendizaje, mejorar los logros de aprendizaje y calidad de la educación incorporando métodos avanzados de enseñanza, así como impulsar la reforma de los sistemas educativos" (UNESCO, 2009, p. 9).

\section{Método}

Esta investigación se realizó con enfoque cualitativo, empleando como método el estudio de casos que, al igual que otros tipos de investigación cualitativa, como la fenomenología, etnografía, grounded theory, investigación narrativa (Creswell, 2007), tiene, entre otras, las siguientes características: está enfocada en el significado y la comprensión; describe y comprende una realidad; conoce cómo las personas participantes en el estudio interpretan sus experiencias y construyen su realidad; quien investiga es el instrumento principal para la colección y análisis de datos; se sigue un proceso inductivo, y presenta riqueza en la descripción (Merrian, 2009).

La atención se centra en el caso en sí, que puede ser una persona, una institución, un programa, política o sistema (Simons, 2011). En general, un estudio de caso es una investigación fáctica que estudia un fenómeno dentro de un contexto real.

Se eligió el método de investigación con estudio de casos con la intención de explorar y analizar cómo se lleva a cabo el proceso de integración de las TIC para favorecer la competencia transversal del Plan de Estudios de la Licenciatura en Educación Primaria 2012 (SEP, 2012). 
doi: http://doi.org/10.15359/ree.24-1.10

URL: http://www.una.ac.cr/educare

CORREO: educare@una.cr

Así mismo, de acuerdo con Rodríguez, Gil y García (1996), se elige un estudio de casos como método de investigación por su carácter crítico, "en tanto que el caso permita confirmar, cambiar, modificar o ampliar el conocimiento sobre el objeto de estudio" (p. 95). La investigación se llevó a cabo en una Escuela Normal del Estado de México. Cuenta con una matrícula de 574 estudiantes; 30 docentes, tres de quienes fungen como personal directivo y siete cubren un interinato por tiempo limitado.

\section{Participantes}

Para esta investigación se realizó un muestreo por conveniencia, se seleccionó a 13 de los 30 docentes de tiempo completo de la escuela normal rural "Lázaro Cárdenas del Río". Como criterio de selección se tomó en cuenta que fueran de diferentes perfiles profesionales y funciones académicas (docentes de inglés, matemáticas, práctica profesional, planeación educativa) para obtener variados puntos de vista; se consideró su disposición para participar en la investigación.

Así mismo, se les eligió porque utilizan las TIC en sus clases, lo que permitió tener una visión más amplia de cómo se favorece su uso, como una competencia transversal de la formación docente.

\section{Instrumentos}

Se aplicó la observación naturalista en eventos situaciones y comportamientos que se dieron en el contexto de la investigación; así mismo, se llevaron a cabo entrevistas a profundidad con cada una de las personas participantes en el estudio.

\section{Procedimientos}

Para recolectar los datos y obtener la información necesaria para dar respuesta a las preguntas de investigación se aplicaron las entrevistas a profundidad a cada participante, las cuales fueron grabadas y transcritas para el análisis de la información (ver Apéndice A). La observación naturalista se llevó a cabo en dos sesiones de clase de cada una de las personas participantes. La información recabada durante las observaciones se registró como notas de campo en un diario. Las respuestas a las entrevistas se grabaron y posteriormente se transcribieron y organizaron por categorías de análisis.

\section{Análisis de datos}

Los datos recolectados se organizaron por categorías: uso de las TIC por parte del personal docente; aprendizaje de las TIC por parte de los maestros y las maestras; aplicación de las TIC en 
el aula; desafíos que implica el uso de las TIC en la formación inicial de docentes; actitudes del estudiantado cuando se emplean las TIC en clase; empleo de las TIC por parte del estudiantado normalista. Se empleó codificación abierta, mediante el programa Atlas.ti, para facilitar su análisis (Valenzuela y Flores, 2013) y dar respuesta a las preguntas de indagación. Para validar la confiabilidad del estudio se utilizó el método de triangulación de datos, comparando la información recabada mediante la observación con los datos aportados durante las entrevistas, así como con los aportes teóricos que dan sustento a este estudio.

\section{Resultados y discusión}

\section{Uso de las TIC por parte del personal docente}

En primer lugar, se observó el nivel de conocimientos con que cuentan el cuerpo docente formador de maestros y maestras y cómo adquirieron dichos conocimientos. En segundo lugar, se indagó si, efectivamente, la aplicación pedagógica de las TIC está estrechamente relacionada con su nivel de conocimientos o dominio (Suárez et al., 2013). A continuación, se presentan los resultados en la Tabla 1.

Tabla 1: Niveles de conocimientos del profesorado en el uso de las tecnologías, de acuerdo con sus propias percepciones

\begin{tabular}{ll}
\hline Nivel & Conocimientos, habilidades y aptitudes \\
\hline Nivel básico & $\begin{array}{l}\text { Manejo de computadora, Laptop, video proyector. Funciones básicas del sistema operativo } \\
\text { Windows. Uso de aplicaciones básicas de Microsoft Office (Word, PowerPoint, Excel). Conocimiento } \\
\text { y uso limitado internet, redes sociales, correo electrónico. }\end{array}$ \\
& $\begin{array}{l}\text { Manejo de computadora, Laptop, video proyecto, tableta, teléfono celular. Redes sociales, } \\
\text { blogs, wiki. Conocimiento y uso de dos o más sistemas operativos. Mayor conocimiento y } \\
\text { uso de Microsoft Office (Word, PowerPoint, Excel). Formato de textos, herramientas de diseño, } \\
\text { funciones de cálculo avanzadas. Internet. Correo electrónico. Conocimiento y aplicación de } \\
\text { recursos informáticos en línea, software educativo, herramientas de productividad, búsqueda } \\
\text { de información empleando buscadores especializados. }\end{array}$ \\
Nivel experto & $\begin{array}{l}\text { Especialista en tecnologías de la información y la comunicación. manejo de computadoras, } \\
\text { laptop, tableta y otros recursos tecnológicos (hardware y software). Redes sociales, blogs, } \\
\text { wiki. Conocimiento y uso de diversos sistemas operativos. Dominio de Microsoft Office } \\
\text { (Word, PowerPoint, Excel), redes, internet. Conocimiento y uso de herramientas y aplicaciones } \\
\text { de búsqueda de información, diseño, software educativo; recursos informáticos en línea, } \\
\text { plataformas educativas, entre otros. }\end{array}$ \\
\hline
\end{tabular}

Nota: Elaboración propia. 
doi: http://doi.org/10.15359/ree.24-1.10

URL: http://www.una.ac.cr/educare

CORREO: educare@una.cr

En las entrevistas, siete docentes expresan un nivel de conocimiento básico, cinco expresan un nivel medio y solo una persona tiene un nivel avanzado, por lo que se destaca la escasa alfabetización informática que presenta el personal docente.

La mayoría de las personas entrevistadas expresaron que sus conocimientos son básicos o elementales. De acuerdo con su testimonio, únicamente conocen el sistema operativo Windows, algunos programas del paquete Office de Microsoft (Word, PowerPoint, Excel), incluso no todos los recursos y funciones de estos programas. Solo un grupo pequeño utiliza con fines didácticos algunos otros programas o software educativos, como Geogebra, CmapTools y Prezi. Solo una persona reportó dominio avanzado de las TIC, debido a su formación profesional como ingeniero en sistemas computacionales.

\section{Aprendizaje de las TIC por parte de los maestros y las maestras}

Las personas participantes reconocen sus limitaciones en el uso de las TIC. Sin embargo, indican estar conscientes de la importancia de prepararse en su uso. Así mismo, reportan que sus conocimientos sobre el uso de las TIC los adquirieron de diversas maneras: como parte de su formación inicial, tomaron cursos, talleres o diplomados; de manera autodidáctica, por iniciativa propia, por necesidades de trabajo; a través del ensayo y el error; consultando a personas expertas o a público usuario frecuente de las TIC, como familiares y amistades de su entorno; revisando tutoriales en internet y como parte de programas de capacitación continua que ofrece la institución para la que laboran. La limitación tecnológica de un grupo pequeño reduce la frecuencia y variedad de recursos utilizados en sus clases.

\section{Aplicación de las TIC en el aula}

El uso de la tecnología es frecuente en los maestros y maestras a la hora de impartir sus clases; las utilizan como recurso didáctico, además motivan al estudiantado a darles este mismo uso con sus alumnos y alumnas de primaria. Coinciden en que es importante incorporar las TIC en la educación, porque además de las ventajas que trae consigo el uso de recursos multimedia hace más entretenidas y amenas las clases.

Los recursos que emplean con mayor frecuencia son la computadora portátil, el video proyector y el internet, por ser estas tecnologías las que más dominan y con las que se cuenta en la escuela normal. Así mismo, en sus clases utilizan el blog, las wikis, las redes sociales, el teléfono celular para enviar y revisar los trabajos de sus estudiantes. Los programas de MicroSoft Office -Word, PowerPoint, Excel- son los que utilizan con mayor frecuencia, en el aula y en trabajos personales. Utilizan programas como Prezi, para hacer presentaciones; software para la enseñanza de la aritmética, algebra, geometría y estadística, como Geogebra. 
Algunas personas plantean que antes de utilizar las TIC es necesario tomar en cuenta el nivel educativo y las características del contexto en donde se van a aplicar; analizar el plan de estudios de educación básica, los programas de los cursos y las habilidades digitales a desarrollar en el estudiantado para potenciar el aprendizaje. Hay docentes con conocimientos de las TIC a quienes se les dificulta aplicarlas en sus clases, lo que representa una debilidad para el desarrollo de la competencia en estudio, pues no se aprovechan las TIC como recursos didácticos en toda su potencialidad. Hacen uso de muy poco software a pesar de que hay una gran cantidad de materiales para fines educativos disponibles en internet de libre acceso.

Las personas entrevistadas coincidieron en que, aunque las TIC no son indispensables para enseñar, sí pueden ser herramientas muy útiles para favorecer el aprendizaje. Consideran necesario prepararse más en este sentido. El personal docente mejor preparado, con mayor dominio de las TIC, sirve de modelo al estudiantado, que aprende al ver cómo sus maestros y maestras aplican los recursos tecnológicos para facilitar su aprendizaje y realizar con mayor eficiencia y eficacia los trabajos académicos.

Profesorado con conocimientos básicos en TIC las usan para exponer con presentaciones elaboradas en PowerPoint y videos relacionados con el tema de la clase. Las escasas competencias digitales del personal docente y la poca variedad de software que utilizan les genera inseguridad y desconfianza al aplicarlas en el aula (Suárez et al., 2010).

Los maestros y maestras que dijeron tener mayor dominio de las TIC incluían, en sus presentaciones, animaciones y sonidos. Hacían uso de software educativo relacionado con el curso que estaban impartiendo y motivaban el estudiantado a elaborar presentaciones de los temas que verían con sus alumnos y alumnas de primaria. Mostraban más confianza al trabajar con las TIC y alentaban a estudiantes a emplearlas con mayor frecuencia en sus prácticas profesionales y no solo como herramientas para elaborar sus trabajos académicos o planificaciones de clases.

\section{Desafíos que implica el uso de las TIC en la formación inicial de docentes}

Las instalaciones del centro de cómputo y los equipos resultan insuficientes para la cantidad de público usuario; aunado a esto, las deficiencias en la conectividad a internet y la carencia software especializado en educación inciden significativamente en la frecuencia de uso de estos recursos, los recursos tecnológicos con que se cuenta son obsoletos y algunos están en malas condiciones.

Para hacer frente a estos retos, las personas participantes, conscientes de la importancia de desarrollar las habilidades digitales del estudiantado, buscan la manera de actualizarse; llevan sus propios equipos de cómputo y video proyectores para dar clases en el aula. Algunos profesores o profesoras utilizan las redes sociales, como facebook o twitter, diseñan blogs o 
doi: http://doi.org/10.15359/ree.24-1.10

URL: http://www.una.ac.cr/educare

CORREO: educare@una.cr

emplean plataformas como Edmodo; aunque no siempre tienen resultados exitosos porque la escuela normal se encuentra en una población rural en donde no existe una adecuada cobertura del servicio de internet. La capacidad de conexión es muy limitada.

Algunos desafíos son:

1. No todo el alumnado tiene el mismo nivel de conocimiento de las TIC.

2. Algunos maestros o maestras manifiestan cierta inseguridad y temor para usar las TIC y sus aplicaciones.

3. La falta de internet en el área académica constituye uno de los principales obstáculos en la búsqueda de información para las actividades educativas, tanto de profesorado como de estudiantes.

4. La falta de infraestructura, equipo y personal especializado en el uso de las TIC limita el desarrollo de habilidades digitales tanto de docentes como de estudiantes.

5. El dominio del inglés representa otro de los desafíos, pues gran cantidad de materiales y recursos educativos para aprender a utilizar las TIC vienen en inglés; así como programas y software educativo de aplicación en asignaturas y cursos educación básica y normal.

\section{Actitudes del estudiantado cuando se emplean las TIC en clase}

Cuando el personal docente utiliza las TIC en sus clases, la mayoría del estudiantado se interesa, pero es mayor su participación en la clase, si se involucra en el diseño de las presentaciones o cuando utiliza las TIC para hacer investigaciones, elaborar sus planificaciones de clases o cuando diseña materiales para trabajar con sus estudiantes de primaria durante sus prácticas profesionales.

Las actitudes del alumnado varían en distintas dimensiones. La totalidad, en menor o mayor medida, hace uso de las tecnologías, principalmente del teléfono celular y de las redes sociales; aunque pocas veces las utiliza para adquirir conocimientos por iniciativa propia. Cuando únicamente observa presentaciones en PowerPoint se distrae, y prefiere tomar fotos con sus teléfonos celulares en lugar de tomar apuntes sobre la explicación que da el maestro o maestra del tema.

Al realizar algún trabajo académico que el profesorado les pide en computadora, generalmente utilizan el procesador de textos Word, pero en la mayoría de los casos sus textos presentan errores, saturación, y solo en pocas ocasiones utilizan herramientas de formato e incluyen audio, video o gráficos. Las ocasiones en que acuden a tomar clases en el centro de cómputo para realizar búsqueda de información en internet relacionada con el programa del 
curso, si el servicio está muy lento, unas pocas personas se muestran aburridas, distraídas, desinteresadas o indiferentes. Por el contrario, se da el caso de que la mayoría del estudiantado manifiesta actitudes positivas de emprendimiento en la elaboración de presentaciones innovadoras y creativas.

\section{Empleo de las TIC por parte del estudiantado normalista}

El alumnado evidencia la adquisición o desarrollo de competencias digitales de diferentes maneras. Se observa un uso cotidiano de las tecnologías para comunicarse o socializar, a través de Facebook, WhatsApp, Twitter. Así mismo, utiliza con bastante facilidad los programas para aprender matemáticas, como Geogebra; elabora mapas conceptuales con CmapTools; presentaciones en Prezi; utiliza buscadores especializados en internet para investigar sobre los temas que abordará en sus clases. Emplea los programas de Microsoft Office, como Word, para la elaboración de sus planificaciones; Excel, para registros de evaluaciones; Publisher, para elaborar boletines y trípticos.

En una clase que el estudiantado presenta, muestra los potenciales de las herramientas digitales, investiga y evidencia pensamiento crítico en revelación de las fuentes de consulta. Busca mostrar sus potencialidades, su creatividad y destreza en el uso de las tecnologías, anticipándose incluso a los retos que enfrentará en un futuro no muy lejano como docentes de primaria; aprende de manera colaborativa, con la guía de sus maestros o maestras, de sus pares o en solitario, en forma autodidacta. Es interesante ver cómo en poco tiempo desarrollan o fortalecen sus habilidades digitales a pesar de las serias limitaciones tecnológicas de la institución, de la falta de recursos económicos y de la desigualdad de oportunidades de acceso a las tecnologías.

\section{Conclusiones}

Esta investigación permitió identificar, como un obstáculo para integrar con mayor éxito las TIC en la educación y potencializar su uso por parte del estudiantado, el hecho de que la mayoría del personal docente considera insuficiente su nivel de conocimiento en TIC. El uso de recursos tecnológicos, como la computadora portátil y el video proyector, así como el empleo de distintos programas de Microsoft Office, como Word, PowerPoint, Excel por parte del personal docente contribuye, de manera significativa, a que el estudiantado aprenda a utilizar estos recursos o fortalezca sus habilidades digitales y emplee dichas tecnologías en la elaboración de trabajos académicos, como reportes o informes de prácticas; planificaciones de clases; presentaciones de distintos temas. El personal docente con mayores habilidades digitales utiliza con mayor frecuencia las TIC, se desempeña con mayor seguridad y confianza en el aula y explica a sus estudiantes cómo usar las TIC con mayor detalle, sugiriéndoles otros programas y recursos tecnológicos para que tengan una mejor preparación y utilicen las tecnologías en sus prácticas de enseñanza en las escuelas primarias en forma exitosa. 
doi: http://doi.org/10.15359/ree.24-1.10

URL: http://www.una.ac.cr/educare

CORREO: educare@una.cr

Un elemento clave en el desarrollo y fortalecimiento de la competencia transversal mencionada fue la actitud del profesorado ante el uso de las TIC en la educación; confianza que, de acuerdo con sus propias percepciones, se tiene por el nivel de conocimientos y habilidades en el manejo de estas tecnologías.

El profesorado normalista con mayores conocimientos en el uso de las TIC, y que utiliza las estrategias didácticas adecuadas durante sus clases, tiene mayores posibilidades de integrarlas con éxito en el proceso de enseñanza-aprendizaje. El profesorado también contribuye a que el estudiantado desarrolle sus habilidades digitales al solicitarles que los informes de sus prácticas profesionales los elaboren con el procesador de textos Word o con otro de su preferencia.

Se encontró que los maestros y maestras motivan a sus estudiantes a utilizar las TIC al solicitarles, como parte de la evaluación de sus cursos, la elaboración de material didáctico en computadora, presentaciones en PowerPoint, mapas conceptuales elaborados con CmapTools.

El estudiantado normalista evidencia la competencia en el uso de las TIC, adquirida en las aulas como parte de su formación como docentes, al aplicar las TIC dentro y fuera de la escuela en sus actividades académicas cotidianas; cuando acude al centro de cómputo y utiliza con cierta destreza las computadoras, el internet, las redes sociales; cuando se prepara para las jornadas de prácticas pedagógicas, cuando busca información para preparar sus clases. La aceptación de la tecnología por parte de los futuros y futuras docentes para su desarrollo personal y profesional es un indicador de la intención de usar las TIC en sus prácticas educativas.

El incremento del número de recursos tecnológicos y el mejoramiento de la conexión a internet ayudaría a fomentar el uso de las computadoras para investigar y buscar información.

Durante el trabajo de campo se tuvieron algunas dificultades, debido a que no se consideraron algunas limitaciones tecnológicas y metodológicas al hacer la investigación, como los problemas de falta de conectividad de internet, que ocasionaron que las clases programadas en el centro de cómputo se tuvieran que posponer; así como la falta de experiencia en el diseño de la investigación y en el análisis de datos cualitativos.

Son varias las implicaciones pedagógicas que tiene esta investigación. Se destaca el impacto positivo de las TIC en el aprendizaje. Estudiantes manifiestan más interés cuando manipulan los recursos tecnológicos para aprender de manera autónoma. Las clases de matemáticas, que generalmente les parecen tediosas, se les hacen interesantes y realizan con buena disposición los ejercicios matemáticos que sus docents les platean. Se observó que experimentan sin temor con distintos programas y aprenden por ensayo y error.

Cabe mencionar que se observó, durante las clases en el centro de cómputo, que una parte del grupo emplea su tiempo consultando sus redes sociales durante la clase, en sus teléfonos celulares, ante la lentitud del servicio de internet, lo que ocasionó que sus docentes se desesperaran. El tiempo de uso es limitado para cada grupo, por lo que a veces tienen que suspender la actividad sin lograr los objetivos de la clase.

16 Sandra Araceli Varela-Ordorica y Jaime Ricardo Valenzuela-González

Los artículos de la Revista Electrónica Educare del Centro de Investigación y Docencia en Educación de la Universidad Nacional, Costa Rica, se comparten bajo términos de la Licencia Creative Commons: Reconocimiento, No Comercial, Sin Obra Derivada 3.0 Costa Rica. Las autorizaciones adicionales a las aquí delimitadas se pueden obtener en el correo: educare@una.cr 
Este estudio permitió descubrir nuevos problemas de investigación respecto de la alfabetización digital de las nuevas generaciones, para lo cual es indispensable partir de la formación inicial de docentes de educación básica, por ello se considera importante plantear las siguientes preguntas de investigación: ¿cómo desde las políticas educativas se puede atender la falta de infraestructura y recursos tecnológicos que inciden, de manera significativa, en el desarrollo de competencias digitales de los futuros personal docente?, ¿de qué manera los organismos e instituciones educativas pueden poner en marcha programas de capacitación, actualización y profesionalización permanentes para el profesorado formador de docentes? Estas inquietudes quedan para nuevos problemas de estudio que permitan formar docentes de primaria con las competencias digitales necesarias para hacer frente a los retos que la época actual demanda.

\section{Referencias}

Almerich, G., Suárez-Rodríguez, J. M., Belloch, C. y Bo, R. M. (2011). Las necesidades formativas del profesorado en TIC: Perfiles formativos y elementos de complejidad. RELIEVE, 17(2), 1-28. doi: https://doi.org/10.7203/relieve.17.2.4006

Almerich, G., Suárez, J. M., Belloch, C. y Orellana, N. (2010). Perfiles del profesorado a partir del conocimiento de los recursos tecnológicos y su relación con el uso que hacen de estas tecnologías. Revista Complutense de Educación, 21(2), 247-269. Recuperado de https:// revistas.ucm.es/index.php/RCED/article/view/RCED1010220247A/15070

Becker, H. J. y Ravitz, J. L. (2001). Computer use by teachers: Are Cuban's predictions correct? Documento presentado en Annual meeting of the American educational research association, Seattle. Recuperado de http://citeseerx.ist.psu.edu/viewdoc/download?doi= 10.1.1.90.6742\&rep=rep1\&type $=$ pdf

Chai, C. S., Koh, J. H. L. y Tsai, C.-C. (2013). A review of technological pedagogical content knowledge. Educational Technology \& Society, 16(2), 31-51. Recuperado de http://www. jstor.org/stable/jeductechsoci.16.2.31

Creswell, J. W. (2007). Qualitative inquiry \& research design: Choosing among five traditions. Thousand Oaks, CA, EE. UU: Sage.

Cuban, L. (2001). Oversold \& underused: Computers in the classroom. Cambridge, Massachusetts: Harvard University Press.

Ertmer, P. A. (2005). Teacher pedagogical beliefs: The final frontier in our quest for technology integration? Educational Technology Research and Development, 53(4), 25-39. doi: https:// doi.org/10.1007/BF02504683

Ertmer, P. A. y Ottenbreit-Leftwich, A. T. (2010). Teacher technology change: How knowledge, confidence, beliefs, and culture intersect. Journal of Research on Technology in Education, 42(3), 255-284. doi: https://doi.org/10.1080/15391523.2010.10782551 
doi: http://doi.org/10.15359/ree.24-1.10

URL: http://www.una.ac.cr/educare

CORREO: educare@una.cr

Ertmer, P. A., Ottenbreit-Leftwich, A. T., Sadik, O., Sendurur, E. y Sendurur, P. (2012). Teacher beliefs and technology integration practices: A critical relationship. Computers \& Education, 59(2), 423-435. doi: https://doi.org/10.1016/j.compedu.2012.02.001

Koh, J. H. L. y Divaharan, S. (2011). Developing pre-service teachers' technology integration expertise through the Tpack-Developing Instructional Model. Journal of Educational Computing Research, 44(1), 35-58. doi: https://doi.org/10.2190/EC.44.1.C

Koh, J. H. L., Chai, C. S. y Tsai, C.-C. (2014). Demographic factors, TPACK constructs, and teachers' perceptions of constructivist-oriented TPACK. Educational Technology \& Society, 17(1), 185196. Recuperado de http://www.jstor.org/stable/jeductechsoci.17.1.185

Merrian, S. B. (2009). Qualitative research. A guide to design and implementation. San Francisco, CA, EE.UU.: Jossey Bass.

Mortera, F. J. (2016). Utilizar tecnologías de la información y de las comunicaciones: La alfabetización digital. En J. R. Valenzuela (Comp.), Competencias transversales para una sociedad basada en conocimiento (pp. 124-147). México: Cengage.

Orellana, N., Almerich, G., Belloch, C. y Díaz, I. (2004). La actitud del profesorado ante las TIC: Un aspecto clave para la integración. Actas del VEncuentro Internacional Anual sobre Educación, Capacitación Profesional y Tecnologías de la Educación, Virtual Educa. Recuperado de https://www.uv.es/bellochc/ute/doc/VE2004 5 6.htm

Riel, M.y Becker, H.J. (2008). Characteristics of teacher leaders for information and communication technology. En J.Voogt y G. Knezek (Eds.), International Handbook of Information Technology in Primary and Secondary Education (Vol. 20, pp. 397-417). Boston, MA, EE.UU.: Springer. doi: https://doi.org/10.1007/978-0-387-73315-9 24

Rodríguez, G., Gil, J. y García, E. (1996). Metodología de la investigación cualitativa. Málaga: Ediciones Aljibe.

Schiro, M. S. (2008). Curriculum theory: Conflicting visions and enduring concerns. Thousand Oaks, CA, EUA: Sage.

Secretaría de Educación Pública. (SEP). (2012). Perfil de egreso de la educación normal. Recuperado de http://www.dgespe.sep.gob.mx/reforma curricular/planes/lepri/plan de estudios/ perfil egreso

Simons, H. (2011). El estudio de caso: Teoría y práctica. Madrid, España: Morata. Recuperado de https://books.google.com.mx/books?id=WZxyAgAAQBAJ\&printsec=frontcover\&hl=es\&s ource $=$ gbs ge summary $r \& c a d=0 \# v=$ onepage $\& q \& f=$ true

Suárez, J. M., Almerich, G., Gargallo, B. y Aliaga, F. M. (2010). Las competencias en TIC del profesorado y su relación con el uso de los recursos tecnológicos. Archivos Analíticos de Políticas Educativas, 18(10). Recuperado de https://epaa.asu.edu/ojs/article/view/755/832 
Suárez, J. M., Almerich, G., Gargallo, B. y Aliaga, F. M. (2013). Las competencias del profesorado en TIC: Estructura básica. Educación XX1, 16(1), 39-62. doi: https://doi.org/10.5944/ educxx1.16.1.716

Suárez, J. M., Almerich, G., Orellana, N. y Belloch, C. (2012). El uso de las TIC por el profesorado no universitario: Modelo básico e influencia de factores personales y contextuales. Revista Iberoamericana de evaluación educativa, 5(1e), 249-265. Recuperado de https://dialnet. unirioja.es/servlet/articulo?codigo $=4526968$

Teo, T. y Noyes, J. (2011). An assessment of the influence of perceived enjoyment and attitude on the intention to use technology among pre-service teachers: A structural equation modelling approach. Computers \& Education, 57(2), 1645-1653. doi: https://doi. org/10.1016/j.compedu.2011.03.002

Tondeur, J., Valcke, M. y van Braak, J. (2008). A multidimensional approach to determinants of computer use in primary education:Teacher and school characteristics.Journal of Computer Assisted Learning, 24(6), 494-506. doi: https://doi.org/10.1111/j.1365-2729.2008.00285.x

Tondeur, J., van Braak, J., Sang, G., Voogt, J., Fisser, P. y Ottenbreit-Leftwich, A. (2011). Preparing pre-service teachers to integrate technology in education: A synthesis of qualitative evidence. Computers \& Education, 59(1), 134-144. doi: https://doi.org/10.1016/j. compedu.2011.10.009

UNESCO. (2008). Estándares de competencia en TIC para docentes. Londres: Autor. Recuperado de http://eduteka.icesi.edu.co/pdfdir/UNESCOEstandaresDocentes.pdf

UNESCO. (2009). Medición de las tecnologías de la información y la comunicación (TIC) en educación - Manual del usuario. Montreal: Autor. Recuperado de http://unesdoc.unesco. org/images/0018/001883/188309s.pdf

Valcke, M., Rots, I., Verbeke, M. y van Braak, J. (2007). ICT teacher training: Evaluation of the curriculum and training approach in Flanders. Teaching and Teacher Education, 23(6), 795808. doi: https://doi.org/10.1016/j.tate.2007.02.004

Valenzuela, J. R. (2016). Capítulo introductorio. En J. R. Valenzuela (Comp.), Competencias transversales para una sociedad basada en conocimiento (pp. 1-27. México: Cengage. Recuperado de http://latinoamerica.cengage.com/ls/competencias-transversales-parauna-sociedad-basada-en-conocimiento/

Valenzuela, J. R. y Flores, M. (2013). Fundamentos de investigación educativa (Vol. 2). México: Editorial Digital del Tecnológico de Monterrey. 
doi: http://doi.org/10.15359/ree.24-1.10

URL: http://www.una.ac.cr/educare

CORREO: educare@una.cr

\section{Apéndice A}

Guion de entrevista

Buenos días, estimado maestro, estimada maestra.

¿Sería tan amable de concederme una entrevista con la finalidad de conocer sus apreciaciones personales sobre el uso de las tecnologías de la información y la comunicación en la educación y cómo utiliza usted dichas tecnologías en sus prácticas educativas?

1. ¿Cómo considera usted que son sus conocimientos en el uso de las tecnologías de la información y la comunicación?

2. ¿Cómo aplica sus conocimientos y habilidades en el uso de estas tecnologías en el aula para propiciar aprendizajes en sus estudiantes?

3. ¿De qué manera hace frente a los desafíos que implica el uso de las TIC en la formación inicial de docentes?

4. ¿Cuáles son las actitudes que manifiestan sus estudiantes cuando emplea las TIC durante sus clases?

5. ¿Qué impacto tiene el uso de las TIC durante sus intervenciones pedagógicas en el desarrollo de la competencia transversal uso de las tecnologías de la información y la comunicación en sus estudiantes?

6. ¿Cómo manifiestan sus estudiantes la adquisición de la competencia uso de las tecnologías de la información y la comunicación, dentro y fuera del aula?

Eso sería todo. ¡Muchas gracias por su participación! 